\title{
VIRTUAL AIS GENERATION MANAGEMENT SYSTEM FOR WATERWAY RISK ASSESSMENT
}

\author{
Jun Sik Kim ${ }^{1}$, Seung Wook Hong ${ }^{2}$ and Suhyun Park ${ }^{3}$ \\ ${ }^{1}$ Division of Computer Engineering, Dongseo University, Busan, Korea \\ ${ }^{2}$ ONE Data Technology, Sasang-gu, Busan, Korea \\ ${ }^{3}$ Division of Computer Engineering, Dongseo University, Busan, Korea
}

\begin{abstract}
The virtual Automatic Identification System (AIS) generation management system is a system for analysing waterway risk by generating virtual AIS data which contains location information of a specific area. The system uses the data as an input data of the IALA Waterway Risk Assessment (IWRAP).
\end{abstract}

\section{KEYWORDS}

AIS, Shipwreck, waterway risk assessment

\section{INTRODUCTION}

The virtual AIS generation management system for waterway risk assessment generates the virtual AIS data which is input data of the program for analyzing risks (running aground, a headon collision) that may occur during navigation of a ship. The generated virtual AIS data includes the position information, and it is possible to assessment the risk at a desired position in the sea. Increasing the number of vessels and expanding the size of vessels may cause the elevation of vessel accidents and because of this human and property damage are becoming serious. Moreover, due to lack of interest in safety consciousness of ship workers (non-compliance with navigation, improper operation of equipment, etc.), the marine safety tribunal ruled out 199 cases $(85 \%$ of 233 cases).

Despite the development of marine technology, marine accidents tend to continue to increase around the world. So, we are making efforts to prevent marine accidents by using various technologies throughout the world. Among them, we are developing a system that uses AIS (Automatic Identification System) data to confirm the situation at the time of the accident. The AIS data can be used to know the type of ship and where the accident occurred, including location information, using the ship's information (ship's size, ship's speed, ship's unique number, MMSI (Maritime Mobile Service Identity) number). Therefore, AIS data can be used to find the place where many ship accidents occur considering the volume and time of shipment, and it can be used to calculate the risk of the shore.

AIS data can be generated by virtually creating data that is based on data assuming accidents that may occur in the territorial waters and enhancing the safety consciousness of the workers. When pioneering a new port waterway, you can create virtual AIS data and perform various simulations of the accident. It can

Dhinaharan Nagamalai et al. (Eds) : CSEIT, NCS, SPM, NeTCoM - 2018

pp. 45-51, 2018. (C) CS \& IT-CSCP 2018

DOI : $10.5121 /$ csit.2018.81804 
predict and prevent marine accidents that may occur in advance. It also uses virtual AIS data in places where it is difficult for the vessel to collect data on a path that does not travel frequently. And if you do not have frequent voyages but want to analyze the risk in your area, you need virtual AIS data generation module.

Virtual AIS can be used as basic data for creating a Korean risk assessment system when the virtual AIS data generation module and the basis data of the risk assessment due to the occurrence of the actual marine accident are provided. The AIS data, which can be used as the basis for prevention of marine accidents, helps to analyse the factors that threaten accidents in the ocean. The virtual AIS data generating management system can collect accident data which is likely to occur in the ocean by virtually creating AIS data used as input data to analyse the waterway risk. The virtual AIS generation management system for waterway risk assessment can analyse the risk beforehand through the virtual AIS data in the case of pioneering a new waterway and to find the location of the new waterway in a high risk area and it can be the foundation of activity.

\section{RELATED RESEARCH}

\subsection{RELATED RESEARCH AIS}

The Automatic Identification System (AIS) is a system adopted by the International Maritime Organization and provides information on ship operations to prevent marine accidents by using two-way data communication between ship-ship, ship-ship and land. The domestic ship automatic identification system is operated in 41 base stations and 13 operating stations. The base station receives the dynamic information, the static information, and the navigation information, which is transmitted from the ship, and transmits the received information to the operating station.

AIS is diverse in equipment depending on the size of the ship, it transmits information every 6 minutes for static information, and time for transmission according to the speed of the ship in case of dynamic information.[2] There are Class A and Class B marine AIS types and SOTDMA (Self-Organized Time Division Multiple Access) methods.[3] SOTDMA is a time division multiple access communication schemes for efficient use of limited communication technologies in the ocean. The operator is able to ask each ship, AtoN, to send information[4].

AIS data consists of 27 message types in total. There are security processing messages, location report messages, control messages, and so on. Virtual AIS data messages that can be used in IWRAP should include location information. The location report of the vessel should be classified into Class A equipment and Class B equipment, and Class A equipment must be installed in the international vessel (300tons or more). Class A equipment location reporting message ID is 1, 2 and 3 and the Class B equipment location reporting message ID is 18. In order to generate a virtual message including the ship information and the location information, the message ID 1, 2, 3 and 18 are generated. The location of the ship can be changed through the virtual AIS message and the virtual AtoN can be installed in the dangerous area by generating the message ID 21 as the virtual AIS message.

\subsection{RISK ASSESSMENT PROGRAM IWRAP}

The IALA Waterway Risk Assessment Program (IWRAP) is a model to analyze waterway risks (ship collision, stranding, etc.)[4]. The International Association of Lighthouse Authorities (IALA) was established in July 1957 to provide ship safety and navigation assistance. The IWRAP program can analyse risks such as head-on collision, cross-over collision, stranding and 
uses decoded AIS data. The IWRAP program shows the probability which the ship collision and stranding frequency information will occur annually[5].

The Virtual AIS generation management system for waterway risk assessment is make virtual AIS data. In IWRAP program MK2 is quantitative evaluation model. The MK2 program can be used to quantitatively assess the risk and analyse the risk of a desired area using virtual AIS data. By creating virtual AIS data in areas where area capable of causing marine accidents, marine accidents can be predicted and prevented in advance[6].

\section{VIRTUAL AIS GENERATION SYSTEM}

The virtual AIS generation management system flow for the waterway risk assessment determines the position where the risk analysis is required or assumes the accident, and inputs the information for generating the virtual AIS data information at the corresponding position to generate the virtual AIS. The generated virtual AIS data is input data of the IWRAP program, which is an accident risk analysis program, and analyses the risk of the corresponding location through the location information in the AIS data. In case of high-risk areas, install the virtual AtoN to display the risk and terminate the analysis. If the risk is low, analyse the location of the surrounding area or other area and record the results. The figure1 below is the flow chart of this system.

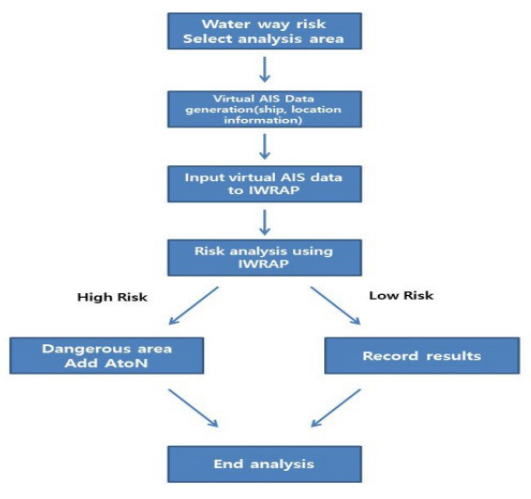

Figure 1. Virtual AIS flow chart.

The Figure 1 to show the flow chart, If you want to analyse the risk in a particular area. First generate some virtual AIS using Virtual AIS generation management System. First, input the ship information (ship type, ship name, MMSI number, latitude longitude). And save AIS data (it is created as a text document). You can input this data to IWRAP program. IWRAP program can check ship traffic. You can analyse the risks based on that.

\subsection{VIRTUAL AIS GENERATION}

The system configuration consists of loading and saving text files through the Files menu, Main, Class A, Class B, AtoN, and Total AIS tabs. The file retrieval function can retrieve a text file, including a separator, a field name, and a space elimination function, and can fetch only a desired portion of various types of text documents by designating a message field sequence number. The import part of the table can be checked in advance before importing the file. Message field specified by the sequence number, it can be confirmed on the Main tab when Open button is pressed. The Main tab identifies the AIS Message Sentence and the ID of the message, and indicates whether the Time Stamp is used or not. When the desired message is clicked, detailed information decoded from the AIS message sentence can be confirmed in the right table. 
The detailed information table differs depending on the message ID. Depending on the size of the vessel, the equipment of the AIS unit is diverse. For ships equipped with Class A equipment, information on specific vessels can be collected based on the desired message ID and Maritime Mobile Service Identity (MMSI) Number in the Class A tab. The position of the vessel can be confirmed by the occurrence of the message according to the time interval, and it can be confirmed through the map. In addition, it is possible to change the marker position information on the map and convert the changed information into AIS data. The Class B tab is a ship equipped with Class B equipment that transmits message IDs 18 and 19, and the contents are the same.

AtoN is a navigational sign that uses light, fluorescence, color, etc. to aid navigation. In the AtoN tab, AIS message ID 21 can be used. It can be displayed when the actual route marking facility is in place, but it is not actual through the Virtual AtoN flag, or in dangerous areas. You can install the AtoN in the corresponding location by checking the hazardous area measured through the Class A and Class B tabs. The installed location is indicated on the map. The Total AIS tab is a tab that allows you to see at a glance which models are culled in Class A, Class B, and AtoN.

\subsection{HOW TO USE SYSTEM MODULE}

The existing AIS data is composed of binary numbers that users cannot recognize. The virtual AIS generation management system can obtain desired information by decoding AIS data composed of binary numbers. The decoded information has a message ID for classifying the AIS message, an MMSI Number for knowing the information of the ship, and the location information including latitude and longitude. It can be used when you want to collect information on existing AIS data before creating a virtual module. In Class A, Class B, and AtoN tabs, you can filter the MMSI number of the vessel you want, and you can see at a glance what signals the vessel is sending. The information of the filtered MMSI Number is displayed on the map and it is easy to know where the signal is transmitted. When you want to create new virtual AIS data, you can display the marker on the map by clicking the desired position on the map.

Since the AIS data that is input data of IWRAP is encoded information, it can generate AIS data through collecting position information on the map by clicking and inputting information such as MMSI Number. By creating AIS data that can send signals to the desired location, you can create virtual AIS data that can analyze the risk through mouse clicks on the map when opening a new route or analyzing the risk in a specific area has. Class A tab can be classified based on message ID numbers 1, 2, and 3, and in the case of Class B tab, based on message IDs 18 and 19, you can easily specify the message ID number and analyze through the AtoN tab. In the case of high riskareas, users can identify dangerous areas. In the Total AIS tab, the virtual AIS data generated from the existing Class A or Class B vessels and the AtoN information generated from the AtoN tab can be displayed on the map to easily locate the dangerous area and the desired area.

When importing existed AIS data, only necessary parts of AIS data can be filtered and recalled, and various types of character encodings can exist, and characters encoded as needed such as UTF-8 and EUC-KR can be analysed. In addition, if new virtual AIS data is generated by clicking on the map, the AIS data generated through the data storage function can be stored in a text form.

You can import new AIS data via Files Button. With the AIS data information imported, you can find the detail information of the ship at total tab. On the Class A tab, the ship's location information is displayed on the map. The position information of the vessel indicated by the marker can be moved anywhere. If you moved markers, it changed location information and make new AIS data. All tabs have 'generate AIS' button on the left down side. You can input the information virtual MMSI number, message ID, SOG, Longitude, Latitude, UTC Hour, UTC Minute, Time stamp and so on. The generate AIS button creates the first new AIS data. Create 
new AIS data by clicking on the map, based on the generated AIS data. If you want to know the location information by vessel, it will be displayed on the map by filtering function. When you save the created data, it is provided as a text document. This text document can analyse dangerous areas with input data from the IWRAP program.

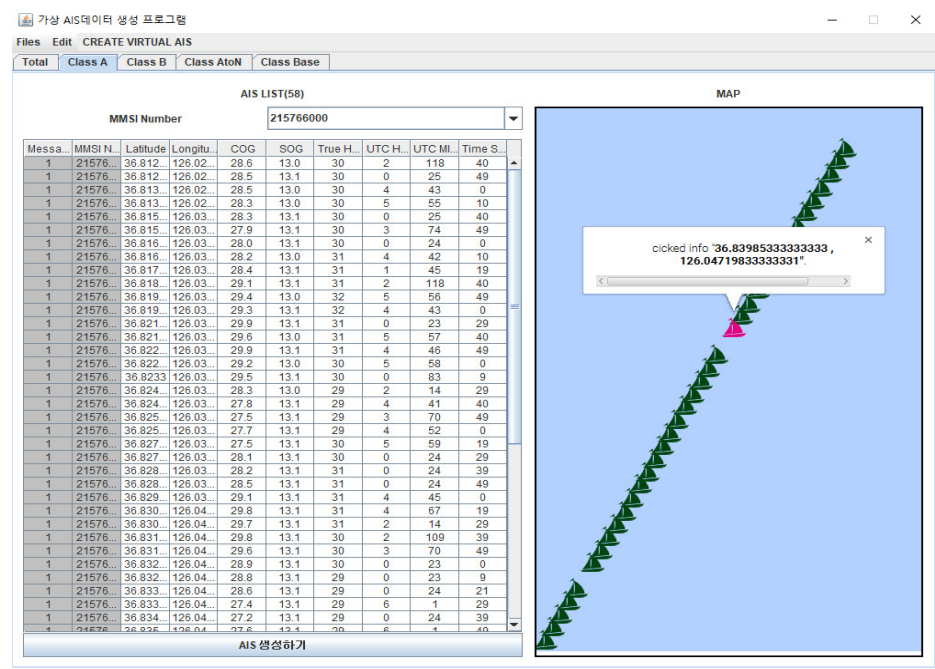

Figure 2. Input AIS data decoding \& check their location

This Figure2 illustrates some information on AIS data from the map. We provide the map of AIS data location information. It can include their longitude and latitude. So we can check their location using the map. If you want make some Virtual AIS, you can create the desired region by clicking on it.

\subsection{RELATIONSHIP BETWEEN VIRTUAL AIS AND IWRAP}

IWRAP can measure the risk of waterways using AIS data as an input data. The Class A and Class B tabs of this system can arbitrarily modify the position and information of the ship to create AIS data and move to the desired position. It can be encoded as AIS data and used as an input data of IWRAP. So, after creating the vessel information and parking the vessel in the aimed area, the risk can be checked by measuring the risk around the radius of the located area through the IWRAP program. The system can generate the virtual AIS data which is needed to assessment the waterway risk and display the risk by virtually locating the AtoN in the dangerous area.

Through the virtual AIS data, IWRAP, which analyse the incident based on the actual ship AIS data can be analysed. When a new route is opened, there is no AIS data because there is no ship movement. Therefore, it is difficult to analyse the risk. Also, the problem can be solved by creating virtual AIS data at the corresponding position.

\section{Conclusions}

This system is dedicated to generate virtual AIS data in order to analyze the waterway risk. It is possible to analyze the risk at a desired location by generating virtual AIS data which can continuously send signals at the waterway location which is the movement path of the ship. AIS data is generated as input data of IWRAP which is a risk analysis program. Rather than path data, by generating virtual AIS data that includes desired location information, the risk can be measured in advance at the location. Measured data does not only indicate simple risk but also it 
establishes a virtual AtoN so that dangerous areas can be displayed in advance even when trying to create a new route. The virtual AIS data generated through the virtual AIS generation management system can assume the accidents that may occur in the ocean and can be used as simulation-based data for analyzing the waterway risk in advance. By creating virtual AIS data, it can play a new role in raising safety awareness of marine casualties and paving the way for new routes.

Risk assessment can be done beforehand through the IWRAP program using virtual AIS data. By measuring the risk at the desired location through the virtual AIS data, the optimal route can be virtually created. The installation of actual navigation aids requires a great deal of cost and time. Therefore, there are many difficulties in changing the route to measure the risk. By creating virtual AIS data time and money can be economized. Through creating the virtual AIS data at the desired location, it can be functioned as the base data to measure the risk in waterway. The virtual AIS data can analyse accidents that may occur on the same line as a collision accident. The AIS data also includes location data, so that it is possible to use the information that can be measured in a ship or a buoy other than an accident, so location information can be provided for observation. The currently generated AIS data is representative of Class A ships, Class B ships, and base station. It will further clarify the information generated and make it possible to collect additional information about the speed and direction of the ship, such as those generated by the actual ship. The future direction is to enable AIS messages to decode and generate a total of 27 messages.

\section{ACKNOWLEDGEMENTS}

It was funded by the 'Ministry of Science and ICT' and was supported by the 'National IT Industry Promotion Agency' (S0708-18-1021, Development of a Service Platform for Crowdsourced High Density Bathymetry). It was funded by the 'Korea Meteorological Institute' (AP00001992, Development of service and advance marine weather contents tailored fisheries).

\section{REFERENCES}

[1] Ministry of Maritime Affairs and Fisheries(Korea), Current status of marine accident (2013 2017).

[2] Technical characteristics for an automatic identification system using time division multiple access in the VHF maritime mobile frequency band ITU-R M.1371-5, February 2014, pp. 111-144.

[3] JinHo Park, (2015), Analysis Method for Maritime Accident using Automatic Identification System, Korea University, Seoul.

[4] SangHoey Lee, (2005), The SOTDMA Algorithm Development and Verification for AIS.

[5] KwangIl Kim, (2014) Analysis is of maritime traffic safety in port waterway using IWRAP.

[6] EuiJong Lee, (2018) A study on the correlation between Port waterway risk Based on IWRAP Mk2 and Marine traffic congestion. 


\section{AUTHORS}

Junsik Kim received B.S. degree in computer engineering from Dongseo University, Korea, respectively. $\mathrm{He}$ is currently working toward the M.S Candidate degree in computer engineering at Dongseo University since March 2018. His research interest is Maritime IT research.

SeungWook Hong received B.S, M.S degree in computer engineering from Dongseo University, Korea, respectively. $\mathrm{He}$ is ONE Data Technology Development Team Manger. He is interest in Maritime IT convergence, Mobile application development.

Suhyun Park received PhD in department of computer science from Busan National University, Korea, respectively. She is a professor of Computer Engineering Division at Dongseo University. She is interest in Maritime IT convergence, Artificial Intelligence and Cloud Computing research. 